\title{
Characterization of Lck-Binding Elements in the Herpesviral Regulatory Tip Protein $^{\dagger}$
}

\author{
Finn Bauer, ${ }^{\ddagger}$ Edith Hofinger, ${ }^{\ddagger}, \|$ Silke Hoffmann, ${ }^{\ddagger}, \perp$ Paul Rösch, ${ }^{\ddagger}$ Kristian Schweimer, ${ }^{\ddagger}$ and Heinrich Sticht*, ${ }^{\prime}$ \\ Lehrstuhl für Biopolymere, Universität Bayreuth, 95440 Bayreuth, Germany, and Institut für Biochemie, \\ Emil-Fischer-Zentrum, Universität Erlangen-Nürnberg, Fahrstrasse 17, 91054 Erlangen, Germany
}

Received July 14, 2004; Revised Manuscript Received September 8, 2004

\begin{abstract}
Herpesvirus saimiri encodes a tyrosine kinase interacting protein (Tip) that binds to T-cellspecific tyrosine kinase Lck via multiple sequence motifs and controls its activity. The regulation of Lck by Tip represents a key mechanism in the transformation of human T-lymphocytes during herpesviral infection. In this study, the interaction of Tip with the regulatory SH3 and SH2 domains of Lck was investigated by biophysical and computational techniques. NMR spectroscopy of isotopically labeled Tip(140-191) revealed that the interaction with the LckSH3 domain is not restricted to the classical prolinerich motif, but also involves the C-terminally adjacent residues which pack into a hydrophobic pocket on the surface of the SH3 domain, thus playing a likely role in mediating binding specificity. Fluorescence binding studies of Tip further demonstrate that Tyr127 in its phosphorylated form represents a strong ligand of the LckSH2 domain, indicating the presence of an additional Lck interaction motif. In contrast, Tyr1 14, known to be essential for STAT-3 binding, does not interact with the LckSH2 domain, showing that the tyrosines in Tip exhibit distinct binding specificity. The existence of numerous interaction sites between Tip and the regulatory domains of Lck implies a complex regulatory mechanism and may have evolved to allow a gradual regulation of Lck activity in different pathogenic states.
\end{abstract}

Src-family tyrosine kinases act as signaling molecules in a wide array of cellular activation processes (1). These enzymes share a general common organization: a myristylated N-terminal "unique" domain followed by the regulatory $\mathrm{SH} 3^{1}$ and $\mathrm{SH} 2$ domains and by the kinase domain containing the active site. The $\mathrm{C}$-terminal region contains a regulatory tyrosine which is bound to the $\mathrm{SH} 2$ domain in its phosphorylated form, thereby reducing kinase activity. Activation of Src kinases can be achieved by dephosphorylation of the tyrosine in the C-terminal region and/or the presence of SH3/ SH2 competing ligands. For full catalytic activity, however, dissociation of both $\mathrm{SH} 3$ and $\mathrm{SH} 2$ from the kinase domain is required $(2,3)$. To ensure an effective activation, numerous

\footnotetext{
This work was supported by grants of the Deutsche Forschungsgemeinschaft to H.S. (SFB466, C11) and P.R. (SFB466, A5).

* To whom correspondence should be addressed. Telephone: +49-9131-8524614. Fax: +49-9131-8522485. E-mail: h.sticht@ biochem.uni-erlangen.de.

Universität Bayreuth.

$\S$ Universität Erlangen-Nürnberg.

" Present address: Institut für Pharmazie, Universität Regensburg, 93040 Regensburg, Germany.

${ }^{\perp}$ Present address: Forschungszentrum Jülich, IBI-2/NMR, 52425 Jülich, Germany.

${ }^{1}$ Abbreviations: AANS, acetamido-anilino-naphthylsulfonic acid; $\mathrm{CD}$, circular dichroism; COSY, correlation spectroscopy; CSKH, C-terminal Src-related kinase homology; HMTA, hamster polyomavirus middle T-antigen; HSQC, heteronuclear single-quantum coherence; HVS, Herpesvirus saimiri; Lck, lymphocyte-specific kinase; IAANS, iodo-acetamido-anilino-naphthylsulfonic acid; NOESY, nuclear Overhauser effect spectroscopy; NMR, nuclear magnetic resonance; rmsd, root-mean-square deviation; $\mathrm{SH} 2, \mathrm{Src}-$ homology 2; SH3, Src-homology 3; SH32, SH3-SH2 domain pair; SH3B, SH3 binding; PPII, type II polyproline helix; TFE, trifluoroethanol; Tio, two-in-one protein; Tip, tyrosine kinase interacting protein; TOCSY, total correlation spectroscopy.
}

proteins therefore target Src kinases by a two-domain interaction with their $\mathrm{SH} 3$ and $\mathrm{SH} 2$ domains. Examples include the activation of Src or Fyn by focal adhesion kinase (4-6), p62 (7), p68 (8), AFAP-110 (9), p130Cas (10), and SIN (11).

For the herpresviral Tip protein that interacts with the lymphoid-specific Src kinase Lck, a variation in the strategy for kinase activation has emerged $(12,13)$. In Tip, a sequence of 37 of the 256 amino acids is necessary as well as sufficient for binding Lck in vitro $(12,14)$. This stretch comprises two binding motifs called CSKH and SH3B $(12,15)$. The SH3B (SH3 binding) motif that matches the consensus of a class II polyproline helix binds to the SH3 domain of Lck (12, 16). The second motif (CSKH, C-terminal Src-related kinase homology) shares some sequence similarity with helix $\alpha \mathrm{I}$ of the kinase domain (12) and was reported to interact with the C-terminal half of Lck (13), but the exact binding site is still unknown.

Tip also contains three conserved tyrosines; phosphorylation of Tyr114 was demonstrated to be required for the activation of STAT factors (17), while the ability of Tip to interact with Lck via phosphorylated tyrosines remains to be investigated. Interestingly, tyrosine phosphorylation was shown to create an LckSH2 domain interaction site in the Tip homologue Tio from Herpesvirus ateles (18), suggesting that the existence of an LckSH2 interaction motif might represent a common feature of herpesviral regulatory proteins.

To gain a better understanding of the interaction between Tip and the regulatory domains of Lck, we have characterized the binding of Tip(140-191) and of different phospho- 


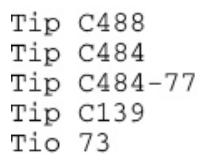

80

NTSS----SSGSDHVPVPIEVNVI IQNSSRTEDELQNS---------KEIE-LTGFQGKI DTSS----SSSSDHVPAPIEVNVIIQNSSRTEDELQNSTKFAVANEGKEIE-LTGFOGKL DTSS--

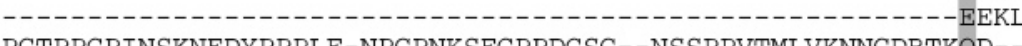
PGTPPGPINSKNEDYPPPLE-NPGPNKSEGPPDGSG--NSSPPVTMLVKNNGDRTKQD--
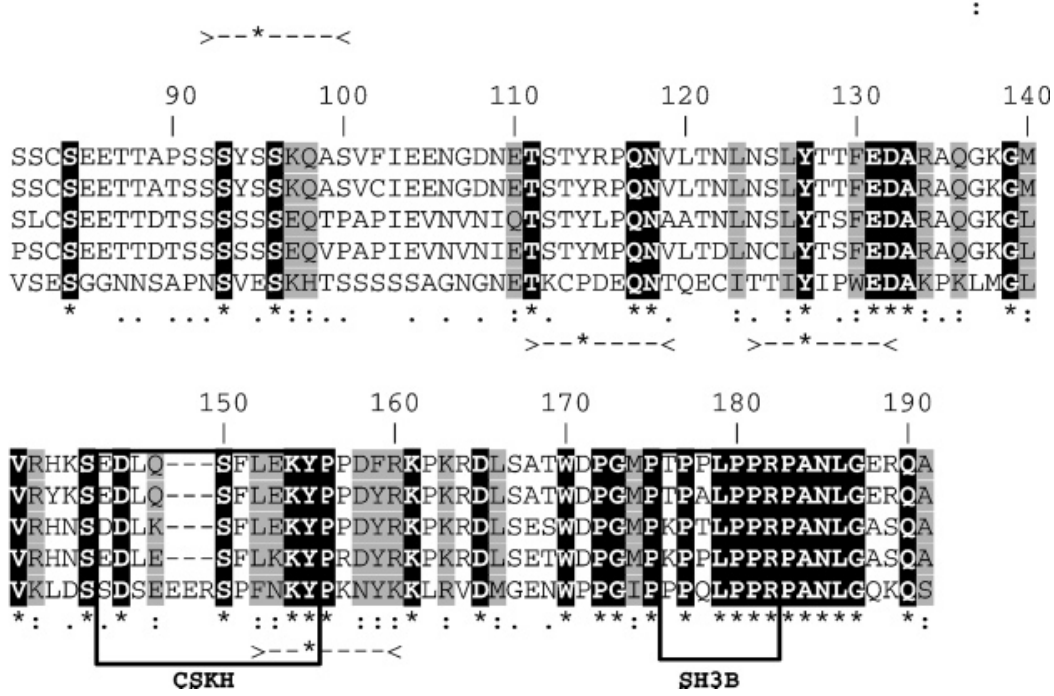

FIGURE 1: Sequence alignment of Tip proteins from different $H$. saimiri subgroup $\mathrm{C}$ strains and of Tio from $H$. ateles strain 73 . Black and gray bars denote strictly and moderately conserved amino acids, respectively. Black boxes highlight the sequence motifs (CSKH and SH3B) that are important for Lck interaction (12). The length and location of the phosphotyrosine peptides are given at the bottom ( $\left.>{ }_{-} *_{-}--_{-}<\right)$. The numbering scheme of Tip C488 is given at the top.

tyrosine-containing Tip peptides by NMR and fluorescence spectroscopy. The aim of this study is to identify those amino acids of Tip that are involved in SH3 binding and to clarify whether the CSKH motif or phosphorylated tyrosines are also able to interact with the regulatory domains of Lck.

Comprehensive characterization of the Tip-Lck interaction is highly desirable in understanding the role of Tip in T-cell growth regulation and of herpesviral pathogenicity. In addition, understanding the regulation of Lck activity by Tip may serve as a basis for the development of new drugs capable of modifying Lck activity in various pathological situations (19).

\section{MATERIALS AND METHODS}

Cloning, Expression, and Purification of Tip(140-191) and Lck. Tip(140-191) was expressed in Escherichia coli BL21(DE3) cells as a ubiquitin-fused construct in a highcopy vector originally engineered by Kohno et al. (20). For ${ }^{15} \mathrm{~N}$ labeling, M9 minimal medium was used with [U-98\% ${ }^{15} \mathrm{~N}$ ]ammonium chloride as the sole nitrogen source. The fusion protein was purified using $\mathrm{Ni}^{2+}$ affinity chromatography (HiTrap Chelating, Amersham Bioscience) utilizing an ÄKTA system (Amersham Bioscience). Fractions containing the pure ubiquitin-Tip(140-191) fusion protein were dialyzed against cleavage buffer and subsequently cleaved using yeast ubiquitin hydrolase (20). Tip(140-191) was separated from ubiquitin and ubiquitin hydrolase by reverse phase HPLC (RP-HPLC) using a Nucleosil 100-7 C18 column (Macherey \& Nagel). The final yields of pure Tip(140-191) were 70 and $30 \mathrm{mg} / \mathrm{L}$ for the expression in LB medium and M9 minimal medium, respectively. Fractions containing pure Tip(140-191) were dried by vacuum centrifugation and stored at $-20{ }^{\circ} \mathrm{C}$.

The SH3 domain of Lck (amino acids 59-119, LckSH3) and the $\mathrm{SH} 3-\mathrm{SH} 2$ domain pair of Lck (amino acids 57-
225, LckSH32) were expressed and purified as described previously $(16,21)$. The purity of all samples was confirmed by SDS-polyacrylamide gel electrophoresis, and the concentration was assessed by measuring $\mathrm{OD}_{280}$ using extinction coefficients calculated from the amino acid sequence.

Peptide Synthesis. All peptides were purchased either from Biosyntan (Berlin, Germany) or from Coring (Gernsheim, Germany) and contained blocked end groups. Unless otherwise stated, sequences of Tip refer to strain C488 of Herpesvirus saimiri and sequences of Tio refer to strain 73 of $H$. ateles. For the fluorescence titration experiments, nine different peptides were used. For the herpesviral ligands, abbreviations in parentheses contain the name of the protein, the sequence position of the phosphorylated tyrosine (Figure 1 ), and an additional letter which indicates point mutations where appropriate: ST(pY)RPQN (Tip114), ST(pY)LPQN (Tip114L), SL(pY)TTFE (Tip127), EK(pY)PPDF (Tip155), ED(pY)PPPL (Tio49), TI(pY)IPWE (Tio136), CQ(pY)QPQP (Lck-COOH, regulatory C-terminus of Lck), and $\mathrm{CQ}(\mathrm{pY})$ EEIP (HMTA, high-affinity SH2 ligand from hamster polyomavirus middle $\mathrm{T}$-antigen). The latter peptide contains a $\mathrm{P} \rightarrow \mathrm{C}$ mutation at the $\mathrm{N}$-terminal sequence position that was used for the attachment of the fluorescence label (see below).

Preparation and Purification of Fluorescent Peptides. Since neither LckSH2 nor the phosphotyrosine peptides that were investigated contain a suitable intrinsic fluorescence probe in the binding region, a fluorophore was attached to the cysteine of the high-affinity HMTA ligand in the $Y-2$ position, allowing competitive fluorescence measurements in which the labeled ligand is displaced from the $\mathrm{SH} 2$ binding site by an unlabeled ligand. The choice of the attachment site and type of the fluorescent probes was based on a previous systematic study (22) which showed that an attachment of acetamido-anilino-naphthylsulfonic acid (AANS) 
at a $Y-2$ position only moderately affects ligand binding to $\mathrm{SH} 2$ domains. Prior to addition of IAANS, the reduced state of the HMTA peptide was assured by addition of equimolar amounts of the reducing agent tris(2-carboxyethyl)phosphine hydrochoride (TCEP) to $100 \mu \mathrm{L}$ of a $1.5 \mathrm{mM}$ sample of HMTA in Tris- $\mathrm{HCl}(\mathrm{pH} 7.5)$. After the mixture had been stirred for $30 \mathrm{~min}$ at $25^{\circ} \mathrm{C}$, aliquots of a $60 \mathrm{mM}$ IAANS stock solution in dimethylformamide were added up to a final concentration of $2 \mathrm{mM}$. The labeling reaction proceeded in the dark for $3 \mathrm{~h}$ at room temperature or overnight at $4{ }^{\circ} \mathrm{C}$. The product was purified by gel filtration using a Superdex peptide HR 10/30 column (Amersham Bioscience).

Fluorescence Spectroscopy. All fluorescence spectra were measured with an F-4500 fluorescence spectrophotometer (Hitachi, Tokyo, Japan) at $21.5^{\circ} \mathrm{C}$ in $1 \mathrm{~cm}$ mirror cuvettes. Initial fluorescence emission spectra were measured for the free and LckSH32-bound AANS-HMTA species (AANSHMTA) after excitation at $330 \mathrm{~nm}$. The maximal difference in the fluorescence emission intensity between free and bound AANS-HMTA was observed at $470 \mathrm{~nm}$; therefore, this wavelength was used for detection in all subsequent titration experiments.

In the first titration experiment, the affinity of AANSHMTA for the LckSH2 domain was determined by gradual addition of small aliquots of LckSH32 from a $65 \mu \mathrm{M}$ stock solution to a $100 \mathrm{nM}$ AANS-HMTA sample up to a final concentration of $2 \mu \mathrm{M}$. The measured fluorescence intensity was properly corrected for sample dilution effects resulting from Lck addition and for minor contributions of the Lck sample buffer to the fluorescence signal. The dissociation constant $\left(K_{\mathrm{D}}\right)$ was calculated assuming a two-state binding model. Since the concentration of the labeled peptide was always low compared to the Lck concentration, the experimental data were fitted according to the standard equation $(16,23)$ :

$$
\Delta F=\frac{\Delta F_{\max }[\mathrm{Lck}]_{0}}{K_{\mathrm{D}}+[\mathrm{Lck}]_{0}}
$$

where $\Delta F_{\text {max }}$ gives the maximal change of the fluorescence amplitude which is observed if all the labeled ligand is bound to Lck. AANS-HMTA bound tightly to LckSH32 $\left(K_{\mathrm{D}}=\right.$ $0.415 \pm 0.030 \mu \mathrm{M})$, showing that the label does not significantly interfere with binding.

Binding affinities of unlabeled $\mathrm{SH} 2$ ligands (U) were determined in a competitive binding assay by gradual addition of $\mathrm{U}$ to a $1.5 \mathrm{~mL}$ sample containing $1 \mu \mathrm{M}$ Lck and $1 \mu \mathrm{M}$ AANS-HMTA and by monitoring the changes in fluorescence at $470 \mathrm{~nm}$. Binding affinities were calculated in a two-step procedure according to the formalism derived by Cheng and Prusoff (24). First, the $\mathrm{IC}_{50}$ value was calculated and is a relative measure of affinity corresponding to a situation in which $50 \%$ of the fluorescence-labeled AANS-HMTA peptide have been displaced from the Lck binding site by the unlabeled $\mathrm{SH} 2$ ligand $\mathrm{U}$.

$$
\Delta F=\frac{\Delta F_{\max }[\mathrm{U}]}{\mathrm{IC}_{50}+[\mathrm{U}]}
$$

Since $\mathrm{IC}_{50}$ is dependent on the concentrations of AANSHMTA and Lck, it provides only a relative measure of affinity. For tightly binding unlabeled ligands, their concentration during the titration experiment is always large compared to their dissociation constant, $K_{\mathrm{D}}^{\mathrm{U}}$. For that case, the dissociation constant can be calculated according to the equation (24)

$$
\mathrm{IC}_{50}=\left(1+\frac{[\mathrm{P}]_{0}}{K_{\mathrm{D}}^{\mathrm{P}}}\right) K_{\mathrm{D}}^{\mathrm{U}}
$$

where $[\mathrm{P}]_{0}$ and $K_{\mathrm{D}}^{\mathrm{P}}$ are the total concentration and dissociation constant of the labeled ligand, respectively.

$C D$ Spectroscopy. Far-UV CD spectra were recorded at $298 \mathrm{~K}$ in $50 \mathrm{mM}$ potassium phosphate (pH 6.4) and $20 \mathrm{mM}$ $\mathrm{NaCl}$ in a $1 \mathrm{~cm}$ cell from 190 to $260 \mathrm{~nm}$ at $20 \mathrm{~nm} / \mathrm{min}$ on a Jasco J810 CD spectrometer at a starting peptide concentration of $5 \mu \mathrm{M}$. Titration experiments were performed by addition of increasing amounts of trifluoroethanol (TFE). The samples were stirred for proper mixing, and the change in peptide concentration as a result of TFE addition was corrected.

NMR Spectroscopy. NMR experiments were recorded on Bruker DRX $600 \mathrm{MHz}$ and DMX $750 \mathrm{MHz}$ spectrometers equipped with pulsed field gradient capabilities at $298 \mathrm{~K}$. Sequential assignment for Tip(144-165) in 30\% TFE was carried out by standard two-dimensional (2D) homonuclear ${ }^{1} \mathrm{H}$ NMR techniques (COSY, TOCSY, and NOESY). For the sequential assignment of the ${ }^{1} \mathrm{H}^{\mathrm{N}},{ }^{1} \mathrm{H}^{\alpha}$, and ${ }^{15} \mathrm{~N}$ resonances of the SH3-bound form of Tip, three-dimensional (3D) HNHA, ${ }^{15} \mathrm{~N}$ TOCSYHSQC, and NOESYHSQC experiments were conducted. Additional side chain resonances were assigned from 2D COSY and $\omega_{2}{ }^{15} \mathrm{~N}$-filtered TOCSY and NOESY experiments $(25,26)$.

The exchange rates for the fast exchanging amide protons were measured using a NewMexico-FHSQC scheme (27). To account for the fact that even small differences in $\mathrm{pH}$ can significantly affect the exchange rates, only changes in the exchange rates of a factor of $>2$ were considered to be significant in the interpretation.

Model Calculation. A NMR-based model for Tip(176190) was generated by the following procedure. In the first step, the relative orientation of residues P177-R182 from Tip in the complex was modeled on the basis of the homologous c-SrcSH3-APP12 complex (28) that exhibits an identical sequence for this proline-rich core motif of the ligand. In addition, the structures of Lck and c-Src are also highly similar (backbone rmsd $=1.1 \AA$ ), and neither Lck nor c-Src shows significant structural changes upon ligand binding $(16,28)$. Therefore, an initial model was generated for this region by docking the PPLPPR structure from the APP12 ligand to the unbound Lck, assuming an identical geometry as in the c-SrcSH3-APP12 complex which results in a system without steric clashes. To keep the geometry of the core motif fixed, a total of 128 intermolecular restraints was included in the subsequent calculation. In addition, torsion angles of the proline residues of the PPII helix were restrained $\left[\phi=-75^{\circ}\right.$ and $\left.\psi=145^{\circ}(29)\right]$.

These homology-based restraints together with the experimentally derived distance restraints served as input for a simulated annealing protocol using X-PLOR 3.851 (30). For conformational space sampling, 45 ps with a time step of 3 fs was simulated at $2000 \mathrm{~K}$, followed by slow cooling 
for $80 \mathrm{ps}$ to $1000 \mathrm{~K}$, and cooling for $60 \mathrm{ps}$ to $100 \mathrm{~K}$, both with a time step of $2 \mathrm{fs}$. A conformational database term for both backbone and side chain dihedral angles was included in the target function to improve the stereochemical properties of the structure $(31,32)$. After simulated annealing, the structure was subjected to 1200 steps of energy minimization, the final 1000 steps without conformational database potential.

\section{RESULTS AND DISCUSSION}

Structural Features of Tip Prior to Lck Binding. The CD spectrum of Tip(140-191) is dominated by a negative band with a minimum at $203 \mathrm{~nm}$ indicating the existence of a type II polyproline (PPII) helix $(33,34)$ that has an intense minimum at $204 \mathrm{~nm}$ (Figure 2A). The wavelength and intensity of this band are highly similar to those detected previously in the shorter Tip(168-187) peptide (16) and thus can most likely be attributed to the proline-rich SH3B motif (Figure 1). As a result of this preformed PPII helix, the entropic cost of the Tip-Lck interaction should be highly reduced and the interaction kinetics should be accelerated (35).

Evidence for the existence of additional elements of secondary structure in Tip(140-191) was obtained from gradual addition of trifluoroethanol (TFE, Figure 2A). TFE can be used as a probe to explore the existence of marginally stable elements of secondary structure, especially $\alpha$-helices (36). Stepwise addition of up to $30 \%$ TFE to Tip(140-191) decreases the negative molar ellipticity at $222 \mathrm{~nm}$ from -970 to $-2200 \mathrm{deg} \mathrm{cm}^{2} \mathrm{dmol}^{-1}$, indicating the presence of a marginally stable $\alpha$-helical secondary structure (Figure 2B) which was not observed previously in the shorter Tip(168191) peptide (16).

This helical structure is localized in the N-terminal half of Tip(140-191) since the shorter Tip(144-165) shows the same tendency as Tip(140-191) to adopt a helical structure, as is evident from the significant increase in the negative ellipticity at $222 \mathrm{~nm}$ in a titration experiment (Figure 2B). The exact location of the $\alpha$-helix was determined by NMR spectroscopy on the basis of the helix-typical NOE pattern (Figure 2C). The presence of medium and strong $\mathrm{HN}-\mathrm{HN}$ NOEs for the stretch from residue 146 to 154 indicates that this helix is formed within the CSKH motif ( ${ }^{146} \mathrm{EDLQS}$ FLEKY ${ }^{155}$ ). This finding is consistent with previous theoretical considerations in which the CSKH motif was thought to bind in a helical conformation (12). The observation that peptide ligands exhibit reduced stability of $\alpha$-helices prior to binding (Figure 2C and Supporting Information) and the stabilization of these elements upon binding were also frequently observed for other systems, including the argininerich $\mathrm{N}$ and Nun proteins from bacteriophages in which their helical structure is stabilized upon RNA binding $(37,38)$. In summary, these results show that Tip(140-191) exhibits an intrinsically marginally stable $\alpha$-helical structure formed by the CSKH motif, and that the polyproline helix of the SH3B motif is preformed prior to Lck binding.

Investigation of the Tip-LckSH3 Interaction by NMR Spectroscopy. First, information about those residues of Tip(140-191) that are affected by LckSH3 binding was obtained from NMR titration experiments (Figure 3). For some resonances (e.g., L179 and L186), it proved to be difficult
A

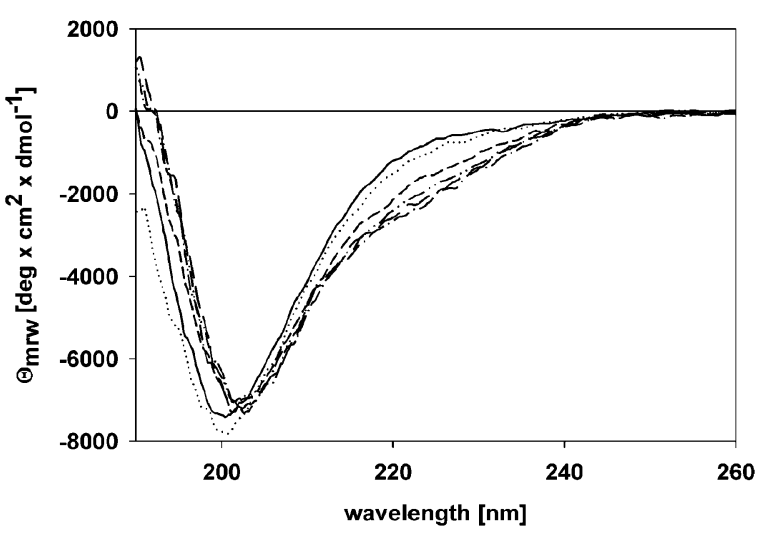

B

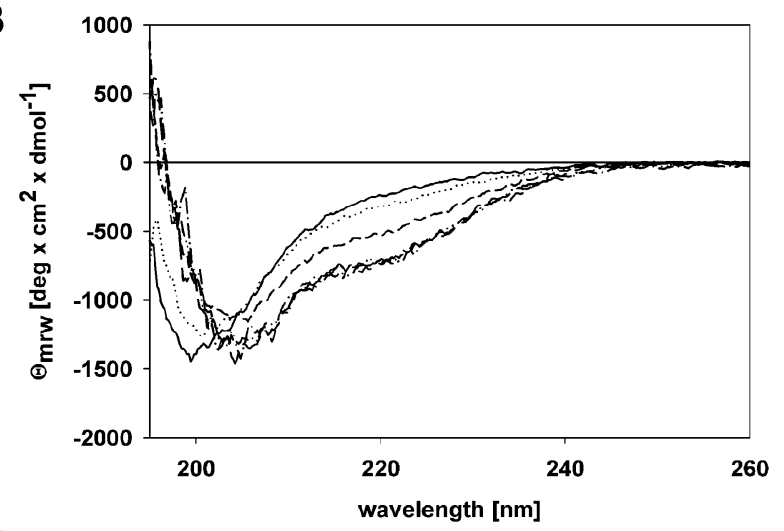

C

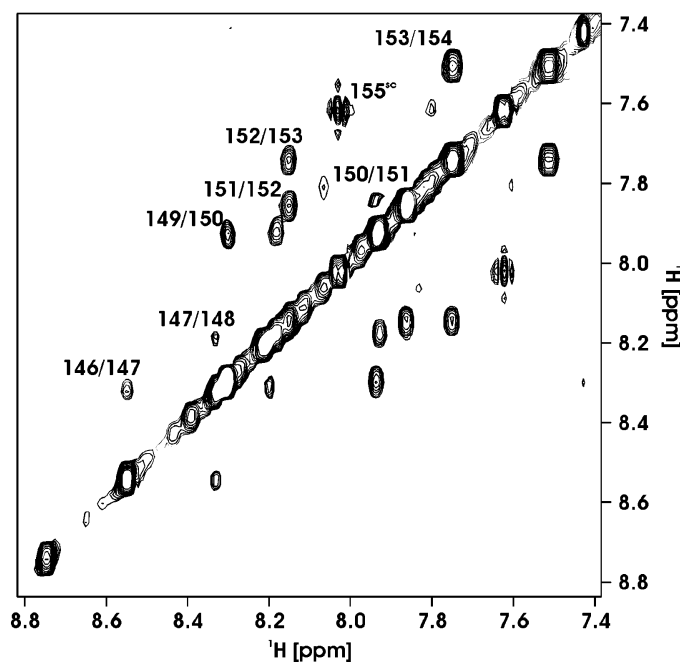

FIGURE 2: Structural features of Tip prior to Lck binding. Far-UV CD spectra of $\operatorname{Tip}(140-191)$ (A) and $\operatorname{Tip}(144-165)$ (B) in the absence $(-)$ and presence of $10 \%(\cdots), 20 \%(--), 30 \%(-\cdot-)$, $40 \%(---)$, and $50 \%(-\cdot-)$ TFE (v/v). Spectra were collected at pH 6.4 with a starting concentration of $5 \mu \mathrm{M}$ peptide. (C) ${ }^{1} \mathrm{H}-$ ${ }^{1} \mathrm{H}$ NOESY spectrum of Tip(144-165) in the presence of $30 \%$ $(\mathrm{v} / \mathrm{v})$ TFE- $d_{2}$ at $\mathrm{pH} 6.0$ and $298 \mathrm{~K}$. Sequential $d_{\mathrm{NN}}(i, i+1){ }^{1} \mathrm{H}-{ }^{1} \mathrm{H}$ NOEs and intraresidual side chain (sc) resonances are labeled with the corresponding sequence position.

to unambiguously identify the cross-peak of the free form most probably resulting from conformational heterogeneity of the unbound Tip. Therefore, resonance assignment was only performed for the Lck-bound form of Tip, and a complete sequence-specific assignment was possible for all residues (except prolines) for which changes in their chemical shifts were observed in the titration experiment.

Significant chemical shift changes (Figure 3) were detected for the residues of the proline-rich sequence motif, T176, 


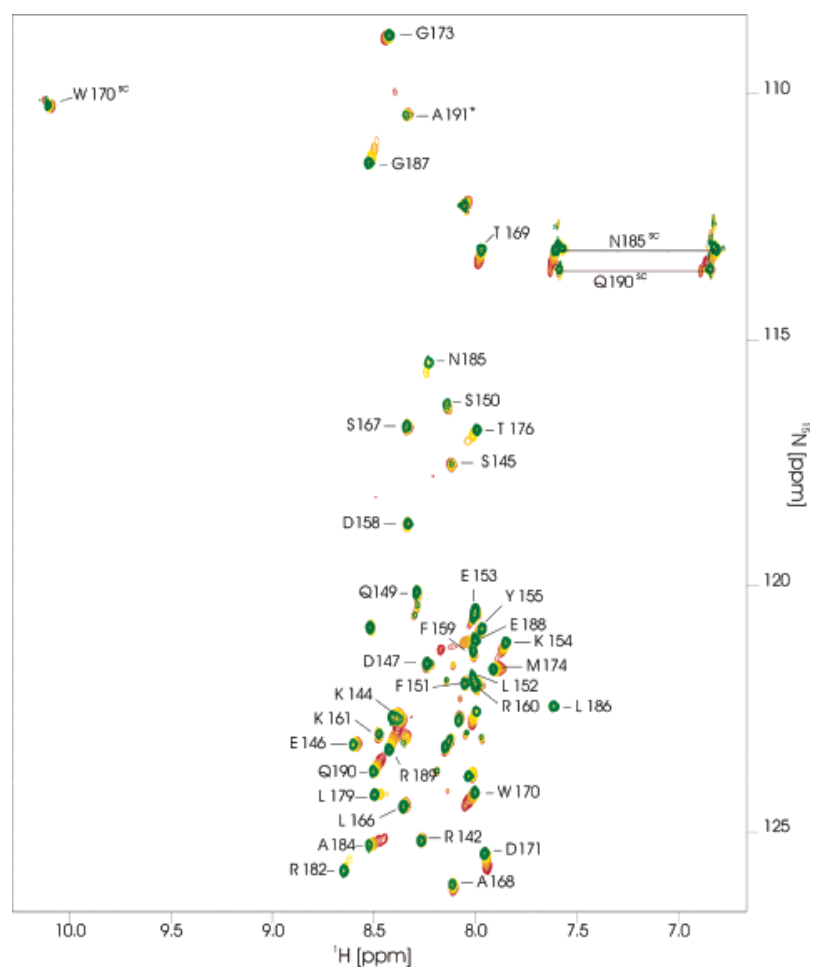

FIGURE 3: NMR titration experiment showing the changes in the ${ }^{15} \mathrm{~N}-{ }^{1} \mathrm{H}$ HSQC spectrum of free Tip $(140-191)$ (red) upon gradual addition of LckSH3. Resonances belonging to the spectra after the final step of the titration are shown in green and are labeled with the corresponding sequence positions. Side chain (sc) $\mathrm{NH}_{2}$ resonances of assigned glutamines and asparagines are connected.

L179, and R182, and for the C-terminally adjacent stretch from A184 to Q190. Minor changes in the chemical shifts were also detected for residues A168-M174, while no significant changes in chemical shifts were observed for residues M140-S167. The same pattern of chemical shift changes in Tip(140-191) was also observed upon gradual addition of the LckSH32 domain pair, revealing that the conserved CSKH motif (E146-Y155) does not interact with the regulatory domains of Lck. This result, together with previous functional studies $(12,13,39)$, now shows that the functionally important CSKH motif exclusively interacts with the kinase domain of Lck, suggesting that Tip actually can be dissected into distinct Lck interaction motifs.

The pattern and magnitude of the chemical shift changes observed for Tip(140-191) are also reflected in the amide proton exchange rates (Figure 4A). Generally, all residues from S167 to A191 exhibit decreased solvent exchange rates for their amide protons after binding to the LckSH3 domain. Although the largest effects are observed for T176 and L179, located within the proline-rich motif, L186-E188 exhibit significant changes, suggesting that they become buried upon complex formation.

More detailed information about the geometry of the TipLck complex was obtained from the analysis of the NOE pattern. On the basis of the CD spectroscopic data, the proline-rich stretch of residues P177-P183 can reliably be assumed to form a polyproline helix, and the small magnitude of the chemical shift changes observed for residues A168M174 suggests that these residues are not directly involved in Lck binding. Thus, the major focus was on the as yet unknown structure of the C-terminal residues of Tip(140191) in the complex.

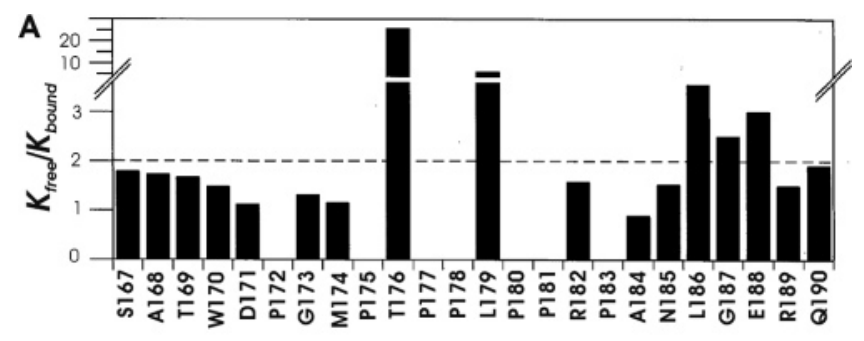

B

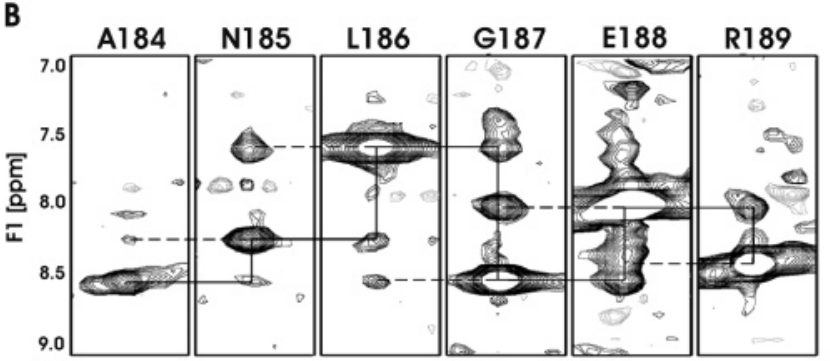

F3 [ppm]

FIGURE 4: Structural features of Lck-bound Tip. (A) Exchange rates of the fast-exchanging amide protons of Tip. The graph gives the ratio of the exchange rates observed in free and Lck-bound Tip. Only changes in the exchange rates of a factor of $>2(--)$ were considered significant in the interpretation. (B) ${ }^{1} \mathrm{H}-{ }^{15} \mathrm{~N}$ NOESYHSQC strip plot of the amide region of Ala184-Arg189 of Tip bound to LckSH3. Sequential $d_{\mathrm{NN}}(i, i+1)$ and $d_{\mathrm{NN}}(i, i-1){ }^{1} \mathrm{H}-$ ${ }^{1} \mathrm{H}$ NOEs are denoted by solid and dashed lines, respectively. The spectrum at $750 \mathrm{MHz}$ of $1.47 \mathrm{mM}\left[\mathrm{U}-98 \%{ }^{15} \mathrm{~N}\right] \mathrm{Tip}(140-191), 1.83$ $\mathrm{mM} \mathrm{LckSH3}, 80 \mathrm{mM}$ potassium phosphate, $35 \mathrm{mM} \mathrm{NaCl}$, and $10 \%$ $(\mathrm{v} / \mathrm{v}) \mathrm{D}_{2} \mathrm{O}$ at $\mathrm{pH} 6.4$ and $298 \mathrm{~K}$.

The strong HN-HN sequential NOEs observed for N185E188 (Figure 4B) indicate that this stretch adopts a helical or turn-like rather than an extended conformation in the complex. Several intermolecular NOEs confirm the existence of contacts between LckSH3 and residues N185-R189 of Tip, but because of their low intensity, an unambiguous assignment was only possible in a few cases. This low intensity most probably results from the dynamics of the complex and was unaffected by several changes in the experimental conditions (e.g., different concentration ratios of Tip and Lck and different temperatures). Nevertheless, some key intermolecular NOEs which are important for the determination of the overall topology of Tip in the complex could be unambiguously assigned between T176 of Tip and F58 of Lck for the N-terminal part and between R182 and G187 of Tip and W40 of Lck for the C-terminal part of the PPII helix, respectively (see the Supporting Information).

The total number of 23 intramolecular and 20 intermolecular NOE distance restraints is too low for an ab initio structure calculation on the basis of NOE distance restraints alone but allows the calculation of an NMR-based model that gives initial information about the location of residues P183-Q190 of Tip in the complex. The set of calculated structures exhibited no single NOE violation of $>0.1 \AA$, showing that the experimental data that are included are in good agreement with the geometry of the PPII helix and with the assumption that the LckSH3 geometry does not change significantly upon complex formation. In the resulting model of the complex, residues A184-E188 are located in a hydrophobic pocket on the LckSH3 surface that is mainly formed by the solvent-exposed hydrophobic side chains of residues W40 and F53 (Figure 5A). The side chain of L186 is placed in the vicinity of the aromatic ring of F53 of Lck, 


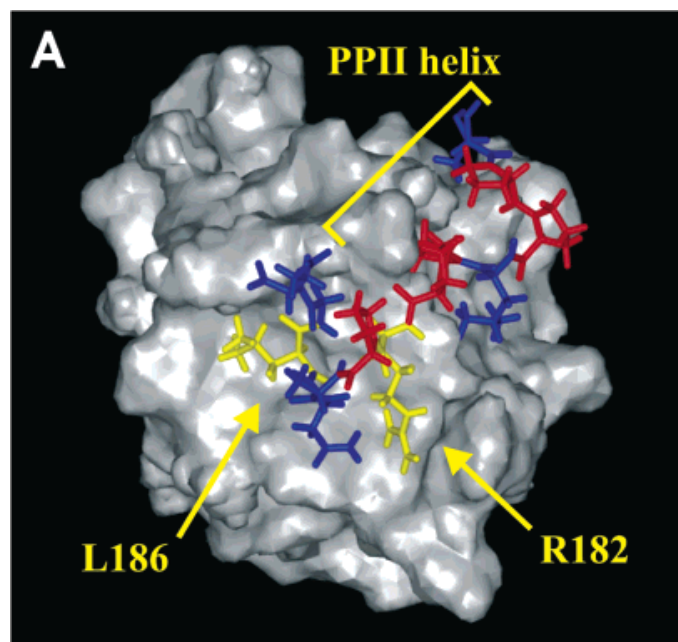

\section{B}
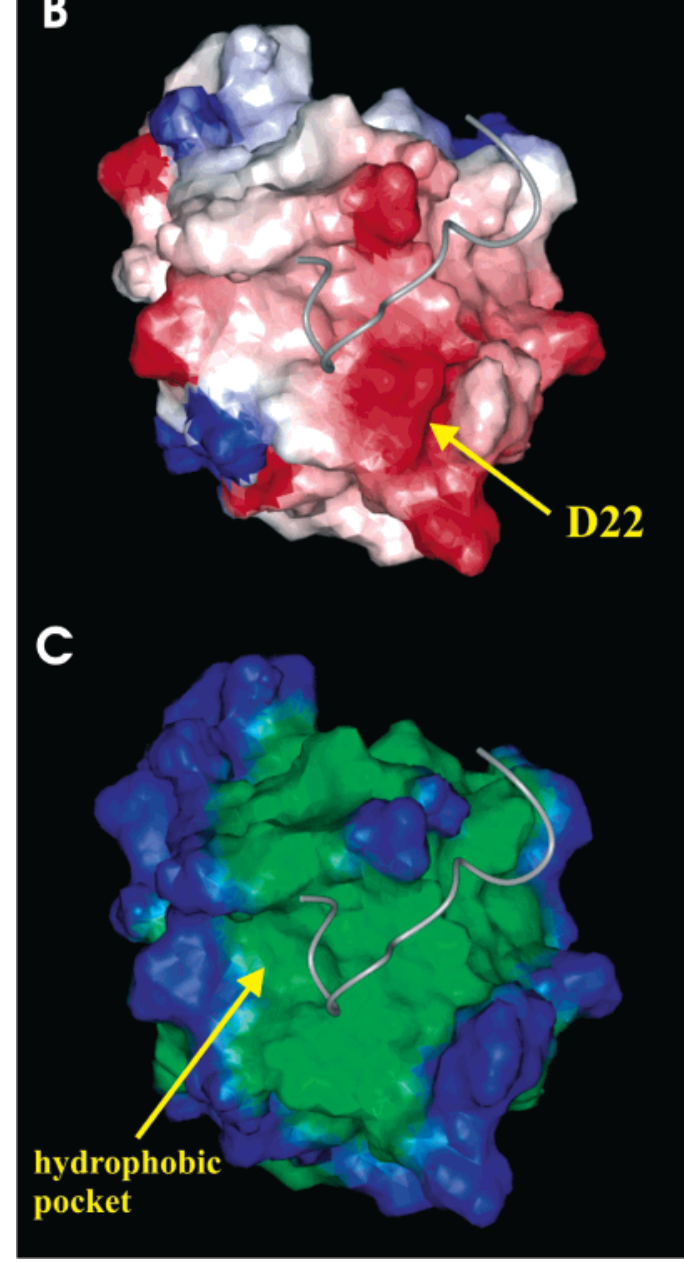

FigurE 5: Model of the LckSH3-Tip complex including the proline-rich "core" motif of Tip and the C-terminally adjacent residues that form an extended binding region. (A) Space-filled representation of the LckSH3 surface (gray) and of residues T176Q190 of Tip in stick presentation. Prolines are colored red, and L186 that packs into a hydrophobic pocket formed by W40 and F53 on the SH3 surface and R182 that builds a salt bridge with D22 are colored yellow and are denoted with arrows. (B) Electrostatic potential of LckSH3. Positively and negatively charged surfaces are colored blue and red, respectively. The high density of negative charge corresponds to D22 which interacts with R182 of Tip, defining the class II orientation of the PPII helix. (C) Hydrophobicity plot of the LckSH3 surface. Hydrophobic and hydrophilic areas are colored green and blue, respectively. CTerminal flanking regions of Tip bind in a helical fashion into a hydrophobic pocket on the LckSH3 surface. providing an explanation for the strong upfield shift of the protons from both methyl groups $(\delta=0.34$ and $0.13 \mathrm{ppm}$, respectively).

In summary, these results from NMR chemical shift and NOE data supplemented by hydrogen exchange experiments point out a distinct role of conserved residues P183-G187 in the Tip-LckSH3 interaction. Thus, it can be concluded that Tip contains an extended binding region consisting of the proline-rich motif itself and of the C-terminal amino acids of this motif that binds into a hydrophobic pocket on the Lck surface (Figure 5C).

The candidate role of this region in the recognition process has been documented previously (28). In this study, peptides that bind in a type I or type II orientation were designed to exploit the hydrophobic pocket for additional contacts. For a peptide that binds in a type I orientation (VSL12), the residues $\mathrm{N}$-terminally adjacent to the proline-rich motif were shown to bind into this pocket and the structure of the respective complex was determined by NMR spectroscopy (28). However, for the APP12 peptide that binds like Tip in a type II orientation, no intermolecular NOEs were observed for the corresponding residues, a fact attributed to the poor packing of the designed region (28). The strict conservation of residues P183-G187 of Tip thus reflects the fact that packing to this hydrophobic pocket has to meet particular steric requirements.

The existence of such "extra" binding regions that are present in addition to the canonical proline-rich motif was also reported for several other $\mathrm{SH} 3$-ligand complexes and frequently plays an important role in the affinity and specificity of complex formation. Interestingly, considerable structural variability in the interaction modes was observed for these extra interaction motifs.

The SH3 domain of Csk specifically recognizes two hydrophobic residues, I625 and V626, in the PEST domain of the SH3-ligand proline-enriched phosphatase (PEP) (40). These two residues are located C-terminally with respect to the conventional proline-rich $\mathrm{SH} 3$ domain recognition sequence of PEP. Unlike in the Tip-Lck system, these residues do not pack into a hydrophobic pocket on the $\mathrm{SH} 3$ surface, but are specifically recognized by A40, T42, and K43 of CskSH3 (40).

In the viral Nef protein that binds to the SH3 domains of various Src-family kinases (3), a specificity pocket was identified, explaining the fundamental differences in the binding specificity observed between HIV and SIV Nef (41). This specificity pocket in Nef is formed by three residues (W113, T117, and Q118) that recognize one single residue of the SH3 domain (R96 in Fyn). Unlike in Tip, these additional residues of Nef are not adjacent to the prolinerich segment in sequence but are spatially proximal to the canonical binding motif in the three-dimensional structure.

The extensive use of a hydrophobic pocket on the LckSH3 surface as observed in the Tip-Lck complex represents a novel variation of the way in which extra interaction motifs in physiological SH3 ligands can recognize their target. The interaction of these flanking residues might play a role in conferring specificity to the interaction or in the proper positioning of the additional interaction motifs present in Tip.

Interaction of Phosphotyrosines in Tip with the SH2 Domain of Lck. The Tip protein from C-subgroup HVS strains contains three conserved tyrosines at positions 114 , 


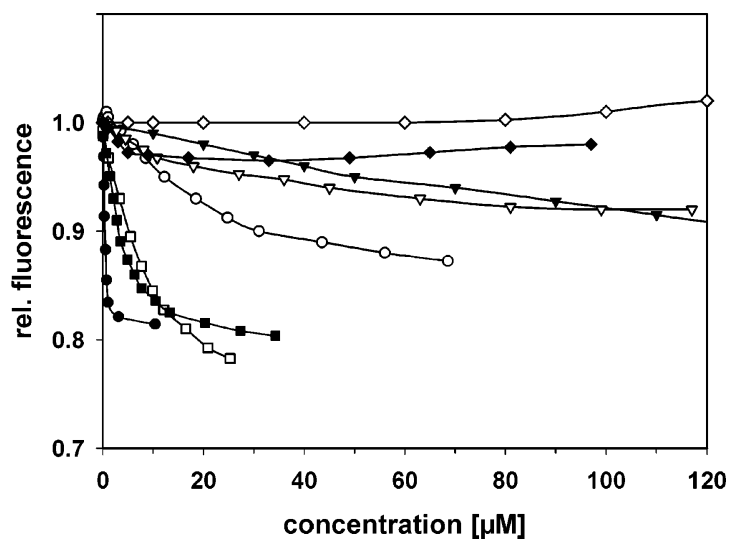

FIGURE 6: Affinity of different phosphotyrosine-containing ligands for the LckSH2 domain. Changes in the relative fluorescence in the competitive binding study in which the AANS-HMTA probe is displaced by one of the following unlabeled phosphotyrosine peptides from the LckSH2 domain: HMTA $(\bullet)$ Lck-COOH $(\bigcirc)$, Tip-114 ( ), Tip-114L $(\diamond)$, Tip-127 ( $\square)$, Tip-155 ( $)$, Tio-49 $(\nabla)$, and Tio-136 (ם). The fluorescence signal was detected at $470 \mathrm{~nm}$, and all intensities were normalized to an identical initial value of 1. Curves were fitted according to the equations given in Materials and Methods. No fit was possible for Tip-114 and Tip-114L, which show no competition in the concentration range of the titration experiment.

127, and 155 (HVS C488 nomenclature). While the Tip homologue Tio from $H$. ateles was shown to interact with the Lck SH2 domain via phosphorylated Y136 (18), no SH2 binding data are yet available for Tip. Therefore, all conserved tyrosines of the H. saimiri $\mathrm{C}$ subtype (Figure 1) were systematically tested for binding, and the two tyrosines from $H$. ateles as well as the $\mathrm{SH} 2$ binding sequences from the Lck C-terminus and the middle $\mathrm{T}$-antigen of hamster polyomavirus served as controls.

Figure 6 summarizes the results of the competitive binding experiments. For peptides Tip-127, Tio-136, and HMTA, asymptotic curves were measured that all approach quite similar relative fluorescence end values $\left(F_{\text {rel }}=\sim 0.8 F_{\max }\right)$, showing that these peptides are able to displace AANSHMTA completely from the binding site. For these peptides that all can be classified as strong binders, $\mathrm{IC}_{50}$ and $K_{\mathrm{D}}$ values were calculated as described in Materials and Methods. The tightest binding was observed for the HMTA peptide, and the measured affinity $\left(K_{\mathrm{D}}=0.095 \pm 0.092 \mu \mathrm{M}\right)$ is within the range of values reported in the literature $\left[K_{\mathrm{D}}=0.14 \mu \mathrm{M}\right.$ for Ac-pYEEI (42); $K_{\mathrm{D}}=0.065 \mu \mathrm{M}$ for EGQpYEEIP (22)].

Tip-127 and Tio-136 exhibit quite similar affinities for LckSH2 of $2.77 \pm 0.18$ and $1.10 \pm 0.12 \mu \mathrm{M}$, respectively. While Y136 of Tio was already reported to be phosphorylated in vivo and to interact with the Lck SH2 domain (18), the ability of Tip-127 to bind strongly to SH2 domains has not been reported. To assess the mechanistic consequences of this result, these affinities were compared to that of the regulatory C-terminus of Lck which is bound to the $\mathrm{SH} 2$ domain in the inactive form of the kinase. For the corresponding Lck-COOH peptide, more than $50 \%$ competition is observed in the binding assay (Figure 6), allowing the calculation of $\mathrm{IC}_{50}$ and $K_{\mathrm{D}}$. As is evident from the shape of the titration curves, Lck-COOH $\left(K_{\mathrm{D}}=8.99 \pm 1.41 \mu \mathrm{M}\right)$ binds significantly more weakly to the SH2 domain than Tip127 or Tio-136, which would in principle render feasible a displacement of this sequence by the herpesviral ligands in vivo. Direct comparison of the affinities appears to be justified by the fact that both types of interactions can be considered as "intramolecular", resulting in similar concentrations of the $\mathrm{SH} 2$ interaction partners. While the C-terminus is covalently bound to Lck, the noncovalent interaction between Tip or Tio and the LckSH3 domain results in a preorganized complex which should already ensure a local proximity of the phosphotyrosine to the $\mathrm{SH} 2$ domain, thus facilitating a replacement of the Lck C-terminus.

For peptides Tip-155 and Tio-49, the $\mathrm{IC}_{50}$ value cannot be determined accurately in the experiment (Figure 6), but can be estimated to be $>120 \mu \mathrm{M}$. This weak interaction is indicative of nonspecific binding and is consistent with the fact that no in vivo phosphorylation and no role in $\mathrm{SH} 2$ binding have been reported for these tyrosines.

Interestingly, Tip-114 that contains an in vivo phosphorylation site and was reported to be important for STAT-3 binding (17) does not interact with the LckSH2 domain at all (Figure 6). This complete absence of interaction is observed for Tip-114 and Tip-114L that originate from HVS strains C488 and C484-77, respectively, and differ by a R $\rightarrow$ L exchange at the $Y+1$ position (Figure 1). Therefore, one can conclude that this absence of the LckSH2 interaction is a conserved property within the C-type group of HVS and may play an important role in reducing the number of nonspecific SH2 interactions to ensure that Y114 is available for binding and activation of STAT-3.

In addition, alternative phosphorylation of Y114 and Y127 might represent a suitable mechanism for switching between two functions of Tip. While phosphorylation at Y127 will function to increase Lck activity, phosphorylation at Y114 is not expected to affect Lck activity itself, but to convert Tip into a STAT-3 activator, thus exerting downstream effects during transcriptional activation.

This complex interaction pattern involving different domains of Lck might have evolved to ensure a gradual regulation of Lck activity, thus controlling T-cell growth and herpesviral pathogenicity. Future functional studies using mutant or chimeric Tip proteins will help in gaining an improved understanding of the in vivo effects of the different types of interactions.

\section{ACKNOWLEDGMENT}

We thank Prof. Franz X. Schmid for providing the opportunity to measure fluorescence spectra in his laboratory and Dr. Brigitte Biesinger and Dr. Armin Ensser for helpful discussions.

\section{SUPPORTING INFORMATION AVAILABLE}

${ }^{1} \mathrm{H}-{ }^{1} \mathrm{H}$ NOESY spectrum of Tip(144-165) in the absence of TFE and ${ }^{1} \mathrm{H}-{ }^{15} \mathrm{~N}$ NOESY-HSQC strip plot of selected residues of Tip bound to LckSH3 showing some intermolecular NOEs. This material is available free of charge via the Internet at http://pubs.acs.org.

\section{REFERENCES}

1. Thomas, S. M., and Brugge, J. S. (1997) Cellular functions regulated by Src family kinases, Annu. Rev. Cell Dev. Biol. 13, $513-609$.

2. Superti-Furga, G., Fumagalli, S., Koegl, M., Courtneidge, S. A., and Draetta, G. (1993) Csk inhibition of c-Src activity requires both the SH2 and SH3 domains of Src, EMBO J. 12, 2625-2634. 
3. Moarefi, I., LaFevre-Bernt, M., Sicheri, F., Huse, M., Lee, C. H., Kuriyan, J., and Miller, W. T. (1997) Activation of the Src-family tyrosine kinase Hck by SH3 domain displacement, Nature 385 , 650-653.

4. Cobb, B. S., Schaller, M. D., Leu, T. H., and Parsons, J. T. (1994) Stable association of pp60src and pp59fyn with the focal adhesionassociated protein tyrosine kinase, pp125FAK, Mol. Cell. Biol. $14,147-155$.

5. Thomas, J. W., Ellis, B., Boerner, R. J., Knight, W. B., White, G. C., II, and Schaller, M. D. (1998) SH2- and SH3-mediated interactions between focal adhesion kinase and Src, J. Biol. Chem. $273,577-583$.

6. Arold, S. T., Ulmer, T. S., Mulhern, T. D., Werner, J. M., Ladbury, J. E., Campbell, I. D., and Noble, M. E. (2001) The role of the Src homology 3-Src homology 2 interface in the regulation of Src kinases, J. Biol. Chem. 276, 17199-17205.

7. Taylor, S. J., and Shalloway, D. (1994) An RNA-binding protein associated with $\mathrm{Src}$ through its $\mathrm{SH} 2$ and $\mathrm{SH} 3$ domains in mitosis, Nature 368, 867-871.

8. Richard, S., Yu, D., Blumer, K. J., Hausladen, D., Olszowy, M. W., Connelly, P. A., and Shaw, A. S. (1995) Association of p62, a multifunctional SH2- and SH3-domain-binding protein, with src family tyrosine kinases, Grb2, and phospholipase C $\gamma-1$, Mol. Cell. Biol. 15, 186-197.

9. Flynn, D. C., Leu, T. H., Reynolds, A. B., and Parsons, J. T. (1993) Identification and sequence analysis of cDNAs encoding a 110kilodalton actin filament-associated pp60src substrate, Mol. Cell. Biol. 13, 7892-7900.

10. Nakamoto, T., Sakai, R., Ozawa, K., Yazaki, Y., and Hirai, H. (1996) Direct binding of C-terminal region of p130Cas to SH2 and SH3 domains of Src kinase, J. Biol. Chem. 271, 8959-8965.

11. Alexandropoulos, K., and Baltimore, D. (1996) Coordinate activation of c-Src by SH3- and SH2-binding sites on a novel p130Casrelated protein, Genes Dev. 10, 1341-1355.

12. Jung, J. U., Lang, S. M., Friedrich, U., Jun, T., Roberts, T. M., Desrosiers, R. C., and Biesinger, B. (1995) Identification of Lckbinding elements in tip of Herpesvirus saimiri, J. Biol. Chem. 270, 20660-20667.

13. Hartley, D. A., Amdjadi, K., Hurley, T. R., Lund, T. C., Medveczky, P. G., and Sefton, B. M. (2000) Activation of the Lck tyrosine protein kinase by the Herpesvirus saimiri tip protein involves two binding interactions, Virology 276, 339-348.

14. Biesinger, B., Tsygankov, A. Y., Fickenscher, H., Emmrich, F., Fleckenstein, B., Bolen, J. B., and Bröker, B. M. (1995) The product of the Herpesvirus saimiri open reading frame 1 (tip) interacts with $\mathrm{T}$ cell-specific kinase p56lck in transformed cells, J. Biol. Chem. 270, 4729-4734.

15. Lund, T., Medveczky, M. M., Neame, P. J., and Medveczky, P. G. (1996) A Herpesvirus saimiri membrane protein required for interleukin-2 independence forms a stable complex with p56lck, J. Virol. 70, 600-606.

16. Schweimer, K., Hoffmann, S., Bauer, F., Friedrich, U., Kardinal, C., Feller, S. M., Biesinger, B., and Sticht, H. (2002) Structural investigation of the binding of a herpesviral protein to the $\mathrm{SH} 3$ domain of tyrosine kinase Lck, Biochemistry 41, 5120-5130.

17. Hartley, D. A., and Cooper, G. M. (2000) Direct binding and activation of STAT transcription factors by the Herpesvirus saimiri protein tip, J. Biol. Chem. 275, 16925-16932.

18. Albrecht, J. C., Friedrich, U., Kardinal, C., Koehn, J., Fleckenstein, B., Feller, S. M., and Biesinger, B. (1999) Herpesvirus ateles gene product Tio interacts with nonreceptor protein tyrosine kinases, J. Virol. 73, 4631-4639.

19. Isakov, N., and Biesinger, B. (2000) Lck protein tyrosine kinase is a key regulator of T-cell activation and a target for signal intervention by Herpesvirus saimiri and other viral gene products, Eur. J. Biochem. 267, 3413-3421.

20. Kohno, T., Kusunoki, H., Sato, K., and Wakamatsu, K. (1998) A new general method for the biosynthesis of stable isotope-enriched peptides using a decahistidine-tagged ubiquitin fusion system: an application to the production of mastoparan- $\mathrm{X}$ uniformly enriched with ${ }^{15} \mathrm{~N}$ and ${ }^{15} \mathrm{~N} /{ }^{13} \mathrm{C}, J$. Biomol. NMR $12,109-121$.

21. Schweimer, K., Kiessling, A., Bauer, F., Hör, S., Hoffmann, S., Rösch, P., and Sticht, H. (2003) Sequence-specific ${ }^{1} \mathrm{H},{ }^{13} \mathrm{C}$ and ${ }^{15} \mathrm{~N}$ resonance assignments of the $\mathrm{SH} 3-\mathrm{SH} 2$ domain pair from the human tyrosine kinase Lck, J. Biomol. NMR 27, 405-406.

22. Cousins-Wasti, R. C., Ingraham, R. H., Morelock, M. M., and Grygon, C. A. (1996) Determination of affinities for lck SH2 binding peptides using a sensitive fluorescence assay: comparison between the pYEEIP and pYQPQP consensus sequences reveals context-dependent binding specificity, Biochemistry 35, 1674616752.

23. Posern, G., Zheng, J., Knudsen, B. S., Kardinal, C., Muller, K. B., Voss, J., Shishido, T., Cowburn, D., Cheng, G., Wang, B., Kruh, G. D., Burrell, S. K., Jacobson, C. A., Lenz, D. M., Zamborelli, T. J., Adermann, K., Hanafusa, H., and Feller, S. M. (1998) Development of highly selective SH3 binding peptides for Crk and CRKL which disrupt Crk complexes with DOCK180, SoS and C3G, Oncogene 16, 1903-1912.

24. Cheng, Y., and Prusoff, W. H. (1973) Relationship between the inhibition constant $\left(\mathrm{K}_{1}\right)$ and the concentration of inhibitor which causes $50 \%$ inhibition $\left(\mathrm{I}_{50}\right)$ of an enzymatic reaction, Biochem. Pharmacol. 22, 3099-3108.

25. Wüthrich, K. (1986) NMR of proteins and nucleic acids, Wiley \& Sons, New York.

26. Cavanagh, J., Fairbrother, W. F., Palmer, A. G., III, and Skelton, N. J. (1996) Protein NMR Spectroscopy: Principles and Application, Academic Press, San Diego.

27. Koide, S., Jahnke, W., and Wright, P. E. (1995) Measurement of intrinsic exchange rates of amide protons in a ${ }^{15} \mathrm{~N}$-labeled peptide, J. Biomol. NMR 6, 306-312.

28. Feng, S., Kasahara, C., Rickles, R. J., and Schreiber, S. L. (1995) Specific interactions outside the proline-rich core of two classes of Src homology 3 ligands, Proc. Natl. Acad. Sci. U.S.A. 92, $12408-12415$

29. Morton, C. J., Pugh, D. J., Brown, E. L., Kahmann, J. D., Renzoni, D. A., and Campbell, I. D. (1996) Solution structure and peptide binding of the SH3 domain from human Fyn, Structure 4, 705714.

30. Brünger, A. T. (1993) X-PLOR, version 3.1, Howard Hughes Medical Institute and Yale University, New Haven, CT.

31. Kuszewski, J., Gronenborn, A. M., and Clore, G. M. (1996) Improving the quality of NMR and crystallographic protein structures by means of a conformational database potential derived from structure databases, Protein Sci. 5, 1067-1080.

32. Neudecker, P., Sticht, H., and Rösch, P. (2001) Improving the efficiency of the Gaussian conformational database potential for the refinement of protein and nucleic acid structures, J. Biomol. NMR 21, 373-375.

33. Viguera, A. R., Arrondo, J. L., Musacchio, A., Saraste, M., and Serrano, L. (1994) Characterization of the interaction of natural proline-rich peptides with five different SH3 domains, Biochemistry 33, 10925-10933.

34. Fasman, G. D. (1996) Circular Dichroism and Conformational Analysis of Biomolecules, Kluwer Academic Publishers, Dordrecht, The Netherlands.

35. Macias, M. J., Wiesner, S., and Sudol, M. (2002) WW and SH3 domains, two different scaffolds to recognize proline-rich ligands, FEBS Lett. 513, 30-37.

36. Buck, M. (1998) Trifluoroethanol and colleagues: cosolvents come of age. Recent studies with peptides and proteins, Q. Rev. Biophys. $31,297-355$

37. Schärpf, M., Sticht, H., Schweimer, K., Boehm, M., Hoffmann, S., and Rösch, P. (2000) Antitermination in bacteriophage $\lambda$. The structure of the N36 peptide-boxB RNA complex, Eur. J. Biochem. 267, 2397-2408.

38. Faber, C., Schärpf, M., Becker, T., Sticht, H., and Rösch, P. (2001) The structure of the coliphage HK022 Nun protein $-\lambda$-phage boxB RNA complex. Implications for the mechanism of transcription termination, J. Biol. Chem. 276, 32064-32070.

39. Friedrich, U. (1999) Src-Kinase Interaktionen des Harpesvirus saimiri Onkoproteins Tip, Dissertation, Universität ErlangenNürnberg, Erlangen, Germany.

40. Ghose, R., Shekhtman, A., Goger, M. J., Ji, H., and Cowburn, D. (2001) A novel, specific interaction involving the Csk SH3 domain and its natural ligand, Nat. Struct. Biol. 8, 998-1004.

41. Collette, Y., Arold, S., Picard, C., Janvier, K., Benichou, S., Benarous, R., Olive, D., and Dumas, C. (2000) HIV-2 and SIV nef proteins target different Src family SH3 domains than does HIV-1 Nef because of a triple amino acid substitution, J. Biol. Chem. 275, 4171-4176.

42. Tong, L., Warren, T. C., King, J., Betageri, R., Rose, J., and Jakes, S. (1996) Crystal structures of the human p561ck SH2 domain in complex with two short phosphotyrosyl peptides at 1.0 and $1.8 \AA$ resolution, J. Mol. Biol. 256, 601-610.

BI0485068 